\title{
SÍNDROME CARDIORRENAL EM CÃES
}

Tayanne Gobbi Mendes'1, Paulo Roberto de Sousa', Rosângela de Oliveira Alves Carvalho².

${ }^{1}$ Mestrando, Programa de Pós-Graduação em Ciência Animal da Escola de Veterinária e Zootecnia da Universidade Federal de Goiás, Goiânia, Brasil. E-mail: taygobbi@hotmail.com.

2 Doutora, Docente da Escola de Veterinária e Zootecnia da Universidade Federal de Goiás, Goiânia, Brasil.

Recebido em: 06/04/2019 - Aprovado em: 10/06/2019 - Publicado em: 30/06/2019 DOI: 10.18677/EnciBio_2019A123

A síndrome cardiorrenal (SCR) ou desordens do eixo cardiovascular-renal (CvRD) é uma afecção que envolve, ao mesmo tempo, o comprometimento renal e cardiovascular. A interação entre esses órgãos é natural e complexa, ambos estão envolvidos em diversos mecanismos vitais para o funcionamento do organismo. A SCR pode ser ocasionada tanto por uma disfunção cardiovascular e renal, em que a disfunção aguda ou crônica em um dos órgãos, pode induzir e perpetuar uma disfunção aguda ou crônica no outro órgão. O diagnóstico da SCR pode ser realizado por meio de biomarcadores renais, cardíacos e exames de imagem, contudo, há a necessidade de identificar quais testes são capazes de detectar precocemente as alterações da doença. O tratamento da SCR é um desafio, pois os pacientes precisam de cuidados que beneficiem os dois sistemas.

PALAVRAS-CHAVE: biomarcadores, cardiorrenal, insuficiência cardíaca.

\section{CARDIORENAL SYNDROME IN DOGS}

\begin{abstract}
A cardiorenal syndrome (SCR) or disorders of the cardiovascular-renal axis (CvRD) is a disease that involves at the same time, renal and cardiovascular impairment. A between between body is natural and complex, both are or serve in organs devices to the working of the organization. SCR can be caused by a cardiovascular and renal dysfunction, that is, an acute or chronic dysfunction in some organ, can induce and perpetuate an acute or chronic dysfunction in the other organ. The diagnosis of SCR can be made by means of renal, cardiac and imaging biomarkers, although all the symptoms are capable of detecting early changes in the disease. Treatment of SCR is a challenge because patients need care that benefits both systems.
\end{abstract}

KEYWORDS: biomarkers, cardiorrenal, heart failure. 


\section{INTRODUÇÃO}

Existe uma interação complexa entre os sistemas cardiovascular e renal, ambos estão envolvidos em diversos mecanismos vitais para o funcionamento orgânico, tais como a regulação da pressão arterial, do tônus vascular, da diurese, a homeostase do volume intravascular e a estabilidade hemodinâmica (SUZUKI et al., 2012). As interações patológicas entre esses dois sistemas de órgãos necessitam de maior definição, classificação e compreensão, uma vez que, a disfunção em ambos os sistemas são as causas mais comuns de morte em cães geriátricos e a lesão primária em um provoca subsequente dano ao outro sistema (RONCO et al., 2010).

A síndrome cardiorrenal (SCR) é definida como distúrbio cardiovascular e renal em que a disfunção aguda ou crônica em um órgão pode induzir e perpetuar uma disfunção aguda ou crônica no outro (RONCO et al., 2010). Ainda são incompreendidos o tempo de início e os mecanismos fisiopatológicos que envolvem a SCR (RONCO; DI LULLO, 2014).

De acordo com o consenso das desordens do eixo cardiovascular-renal (CvRD), a SCR é definida como danos estruturais e funcionais no sistema cardiovascular e renal, induzidos por doenças, toxinas ou substâncias, que podem levar a interações anormais entre esses sistemas (POUCHELON et al., 2015).

A classificação veterinária das desordens do eixo cardiovascular-renal (CvRD) são subdivididas em três grupos: $\mathrm{CVRD}_{H}$ representa a disfunção cardíaca primária culminando em disfunção renal. $\mathrm{CVRD}_{K}$ refere-se a lesão renal primária que propicia o desenvolvimento de disfunção cardíaca. $\mathrm{E}$ a $\mathrm{CvRD}_{\circ}$ que envolve as disfunções cardíaca e renal secundárias a uma alteração sistêmica (RONCO; DI LULLO, 2014; POUCHELON et al., 2015).

O estadiamento clínico, diagnóstico e acompanhamento das doenças do eixo cardiovascular e renal requerem a integração de diversas informações obtidas desde o histórico clínico do paciente até exames complementares e testes diagnósticos (POUCHELON et al., 2015). Novos biomarcadores específicos para a identificação de lesões cardíacas ou renais estão em estudo e apresentam potencial para identificar o desenvolvimento das disfunções e suas interações de forma mais precoce, quando comparados às avaliações clínicas convencionais (ORVALHO; COWGILL, 2017).

O diagnóstico e estadiamento precisos e precoces são essenciais para a detecção da SCR e para o planejamento de planos terapêuticos e estabilização do paciente (POUCHELON et al., 2015). Os novos biomarcadores podem facilitar alvos terapêuticos e o acompanhamento das doenças cardiovasculares e renais, minimizando o estabelecimento da SCR (ORVALHO; COWGILL, 2017).

Contudo, há a necessidade de mais estudos sobre marcadores que são capazes de detectar estágios iniciais da SCR, de forma a possibilitar manejos terapêuticos ainda mais precoces, prevenir o agravamento da doença cardíaca e da lesão renal mútua, aumentando as chances de sobrevida do paciente, assim como a qualidade de vida do mesmo (ORVALHO; COWGILL, 2017).

O conhecimento entre os médicos veterinários sobre as diferentes ferramentas de diagnóstico é indispensável para detectar precocemente a SCR e, consequentemente, instituir um manejo terapêutico adequado (POUCHELON et al., 2015). Nesse sentido, o objetivo deste trabalho, portanto, foi realizar uma revisão da literatura sobre a síndrome cardiorrenal em cães. 


\section{REVISÃO DE LITERATURA}

A insuficiência cardíaca cursa, a curto ou a longo prazo, com a perda da capacidade renal em manter a homeostase. Esta inter-relação de lesão mútua, denominada síndrome cardiorrenal (SCR), já é amplamente estudada na medicina, no entanto, há poucos estudos na medicina veterinária, tornando necessária maior atenção pelos profissionais da área, uma vez que ambas as doenças, cardíacas e renais, estão entre as causas mais comuns de morte em cães geriátricos (RONCO et al., 2010).

As cardiopatias representam $10 \%$ das enfermidades que acometem os cães. Entre a ocorrência das doenças cardíacas, a doença degenerativa valvar crônica está entre as mais frequentes, correspondendo a $75 \%$ dos pacientes com cardiopatia atendidos por veterinários. A doença degenerativa valvar crônica é mais comumente observada na valva atrioventricular esquerda ou mitral, porém em aproximadamente $30 \%$ dos casos a valva atrioventricular direita ou tricúspide apresenta-se envolvida (ATKINS et al., 2009; FREITAS et al., 2016).

A doença renal crônica (DRC) está entre as principais doenças que afetam cães e gatos geriátricos, e apresenta índices significativos de morbidade e mortalidade na rotina veterinária. A prevalência de DRC foi estimada em $0,5 \%$ a $3,74 \%$ em cães, aumentando para cerca de $5,79 \%$ em cães com mais de 12 anos (POLZIN, 2011; O'NEILL et al., 2013).

$O$ dano ocasionado aos néfrons na DRC normalmente é irreversível e progressivo, aumentando mais de cinco vezes as chances de animais acima de 12 anos terem doença renal em comparação a idades anteriores (O'NEILL et al., 2013). Ainda, animais com DRC podem comumente apresentar hipertensão arterial sistêmica (HAS) (WEHNER et al., 2008; KANG et al., 2018). Há estudos que demonstram que a HAS representa $31 \%$ a $51 \%$ dos casos de cães com DRC, considerada uma sequela desta enfermidade (WEHNER et al., 2008).

\section{Definição}

A SCR é definida como uma interação mútua do sistema cardiovascular e renal, no qual a disfunção em um órgão inicia e perpetua uma lesão em outro órgão. Tais interações incluem uma variedade de condições, agudas ou crônicas, onde os efeitos diretos e indiretos do sistema que se apresenta disfuncional, pode prejudicar significativamente a função do outro sistema (RONCO et al., 2010).

\section{Classificação}

As classificações da SCR em humanos e em animais são conceitualmente semelhantes. Ambas descrevem o insulto primário, a sequência de eventos e a cronicidade do processo; mas desconsideram a fisiopatologia e o manejo terapêutico (ORVALHO; COWGILL, 2017; RANGASWAMI et al., 2019).

\section{Classificação da Síndrome cardiorrenal humana}

Para simplificar e identificar o cenário clínico da SCR humana, foram propostos cinco tipos de classificações : (RONCO et al., 2008; RONCO et al., 2010; RONCO; DI LULLO, 2014).

A SCR tipo 1 (Síndrome cardiorrenal aguda) é caracterizada por comprometimento agudo da função cardíaca que leva à lesão renal aguda. Existem diversos fatores que ocasionam o insulto primário, como por exemplo, o choque 
cardiogênico. Neste caso, pode ser observada lesão renal devido a redução da perfusão renal pela falha da bomba cardíaca, ativação de mecanismos neurohormonal e simpático em resposta à congestão renal secundária, diminuição da diurese devido a congestão, o que requer o uso de diuréticos como tratamento, comprometendo cada vez mais a resposta renal (LIANG et al., 2008).

Já a SCR tipo 2 (Síndrome cardiorrenal crônica), evidencia que a doença cardiovascular crônica causa progressiva doença renal crônica (RONCO et al., 2008). A insuficiência cardíaca crônica, por sua vez, provoca redução persistente da perfusão renal e leva a insuficiência renal por congestão venosa ou nefropatia congestiva. São observadas também alterações neuro-hormonais associadas à estimulação simpática crônica (produção de epinefrina, angiotensina, endotelina e liberação de peptídeos natriuréticos e óxido nítrico) (JESSUP; COSTANZO, 2009). O uso de diuréticos e os agentes bloqueadores do sistema renina-angiotensinaaldosterona (SRAA) para o tratamento da insuficiência cardíaca congestiva (ICC), podem causar alterações hemodinâmicas intrarrenais e serem prejudiciais aos rins, devido aos efeitos de hipovolemia e hipotensão (LIANG et al., 2008).

A SCR tipo 3 (Síndrome renocardíaca aguda) é descrita como uma lesão renal aguda primária, que desenvolve disfunção cardíaca secundária. Há vários mecanismos que levam a doença renal a provocar disfunção cardíaca, como a sobrecarga hídrica, distúrbios eletrolíticos, ativação neuro-hormonal e fatores depressores do miocárdio, contribuindo para o desenvolvimento de arritmias, pericardite e insuficiência cardíaca aguda (RONCO et al., 2008).

A SCR tipo 4 (Síndrome renocardíaca crônica), trata-se de uma doença renal crônica primária desencadeando lesão cardíaca secundária. A DRC a longo prazo pode desencadear sequelas ao sistema cardiovascular com a diminuição da função cardíaca, disfunção diastólica, hipertrofia ventricular esquerda e aumento do risco de eventos cardiovasculares adversos (RONCO et al., 2008).

E a SCR tipo 5 (Síndrome cardiorrenal secundária) inclui a doença cardíaca e renal secundária a uma condição sistêmica aguda ou crônica (RONCO et al., 2008; POUCHELON et al., 2015). Entre as condições sistêmicas que provocam danos secundários a outros órgãos, a sepse é a condição aguda mais comum que afeta o coração e os rins (LANGHORN et al., 2013; POUCHELON et al., 2015). A pancreatite e o hiperadrenocorticismo, por exemplo, são doenças crônicas que causam danos no sistema renal e cardiovascular (POLZIN, 2011; WALDUM; OS, 2013).

\section{Classificação das desordens do eixo cardiovascular-renal em animais}

Em 2015, um grupo formado por 13 especialistas na área de cardiologia e nefrologia veterinária da Europa e América do Norte, devido à tamanha demanda e preocupação em aumentar a consciencialização sobre a SCR, criaram um consenso em torno da definição, fisiopatogenia, diagnóstico e recomendações para cães e gatos com doenças no eixo cardiovascular-renal (CvRD) (POUCHELON et al., 2015).

Os CvRD de acordo com o consenso, são definidos como danos morfológicos e funcionais no sistema cardiovascular e renal, induzidos por substâncias ou doenças. Tais alterações provocam mudanças anormais entre esses sistemas e podem propiciar prejuízo de um, ambos ou mais órgãos (POUCHELON et al., 2015). 
A classificação veterinária das CvRD criada para classificar a SCR veterinária tem como base os tipos de classificação humana. São subdivididas em três grupos: $C_{V R D_{H}}$ refere-se à disfunção cardíaca que leva a lesão renal; $C v R D_{K}$ é descrita como a lesão renal que provoca as alterações cardíacas; e a CvRDo que caracteriza a disfunção cardíaca e renal secundária a uma alteração sistêmica aguda ou crônica, como doenças, toxinas ou substâncias. As três categorias ainda podem ser subdivididas em doença estável (S) ou doença instável (U) com base na apresentação clínica do paciente (RONCO et al., 2008; POUCHELON et al., 2015).

CvRD $_{H}$ reporta-se à lesão renal decorrente de um processo primário de disfunção envolvendo o sistema cardiovascular (POUCHELON et al., 2015). Alguns mecanismos com potenciais prejudiciais de $\mathrm{CvRD}_{H}$ incluem a diminuição da perfusão renal secundária à redução do débito cardíaco (HAASE et al., 2013), choque cardiogênico e hipotensão sistêmica, que consequentemente leva a redução da taxa de filtração glomerular (TFG) e ao aumento da creatinina sérica e nitrogênio uréico sérico (BUN) (LIANG et al., 2008). Há casos de congestão renal passiva ocasionada pela insuficiência cardíaca congestiva; distúrbios hemodinâmicos que levam a ativação de sistemas neuro-hormonais como o sistema renina-angiotensinaaldosterona (SRAA) e o sistema nervoso simpático (SNS); alterações de hipoperfusão e hipóxia que propiciam a geração de estresse oxidativo nesses pacientes (HAASE et al., 2013); casos de aumento da pressão arterial sistêmica que provocam doença glomerular; desenvolvimento de tromboembolismo arterial sistêmico pelo fluxo turbulento e estase sanguínea que pode levar ao infarto renal (POUCHELON et al., 2015).

CvRD $_{K}$ refere-se à disfunção cardiovascular que emana de um processo primário de doença renal (POUCHELON et al., 2015). Dentre as alterações que apresentam potenciais prejudiciais de $C_{V R D}$, incluem as anormalidades eletrolíticas, como a hipercalemia e hipocalemia que estão associadas as arritmias cardíacas (POUCHELON et al., 2015; KELLER et al., 2016; BAGSHAW et al., 2018); o uso de medicamentos que são utilizados para o tratamento das doenças cardiovasculares (digoxina, enalapril e atenolol) e que são excretados via renal provocando lesão renal primária, pois quando há disfunção renal e redução da depuração dos fármacos, ocorre o acúmulo das substâncias e sinais de toxicidade farmacológicas, além de desenvolver arritmias, hipotensão e piora da função miocárdica; distúrbios hemodinâmicos, como alterações de volume de fluido, que são sempre anormais em pacientes com doença renal grave e afetam o sistema cardiovascular devido ao baixo débito (POLZIN, 2011).

Outras alterações normalmente observadas e que desenvolvem $\mathrm{CVRD}_{K}$ são a presença de alterações clínicas como hipodipsia, anorexia ou vômitos urêmicos, que podem desenvolver depleção de volume, redução do débito cardíaco e redução da perfusão sistêmica (POUCHELON et al., 2015); casos de hipertensão sistêmica, uma sequela comum à DRC, que pode resultar em hipertrofia ventricular esquerda, disfunção miocárdica (ORVALHO; COWGILL, 2017), aumento da pós-carga, piora da insuficiência mitral ou aórtica, arritmias, vasculopatias e retinopatia; animais que apresentam estado de azotemia, que podem ter efeitos deletérios aos miócitos cardíacos e ocasionar a pericardite urêmica, devido ao aumento exacerbado de substâncias endógenas; ativação do eixo renina-angiotensina-aldosterona levando à retenção de sódio e água, sobrecarga cardíaca e remodelamento cardíaco, vascular ou congestão sistêmica; desenvolvimento de anemia secundária à doença renal 
crônica, ocasionada pela inflamação e déficit de produção de eritropoetina, que desenvolve sobrecarga de volume e redução da oxigenação de tecidos, principalmente o cardiovascular (POUCHELON et al., 2015).

O CVRDo atribui a SCR a lesões ou disfunções renais e cardiovasculares secundárias, provenientes de uma alteração sistêmica primária, seja aguda ou crônica (POUCHELON et al., 2015). As causas que possuem potenciais prejudiciais de $\mathrm{CvRD}_{\circ}$ incluem o estado de sepse, doenças infecciosas, endocrinopatias, pancreatite (POLZIN, 2011), neoplasias, amiloidoses, dilatação vólvulo gástrica, entre outros (POUCHELON et al., 2015).

\section{Fisiopatogenia}

À medida que as lesões valvares progridem, a insuficiência cardíaca tornase clinicamente significativa, provocando distúrbios hemodinâmicos e interferindo no aporte sanguíneo para órgãos alvo, podendo iniciar ou perpetuar lesão aguda ou crônica, como exemplo, as lesões renais (ORVALHO; COWGILL, 2017). Há diversos mecanismos compensatórios que são importantes para a manutenção do organismo e que envolvem os sistemas cardiovascular e renal. Tais mecanismos mantém o equilíbrio do organismo atuando na regulação da pressão arterial, tônus vascular, diurese, homeostase do volume intravascular e estabilidade hemodinâmica (SUZUKI et al., 2012).

A redução da função renal associada a perda progressiva da função cardíaca, desencadeia a ativação de sistemas neuro-hormonais, e consequentemente favorece o remodelamento cardíaco ( COWGILL et al., 2016; ORVALHO; COWGILL, 2017). Foi observado em um estudo, onde foi realizada a indução da síndrome cardiorrenal em cães, que as interconexões do SRAA, o SNS e vasopressina encontram-se envolvidas de uma maneira complexa nesses pacientes, porém ainda não é clara a patogênese exata dessas alterações na referida síndrome (SUZUKI et al., 2012). A disfunção renal com subsequente azotemia em pacientes com insuficiência cardíaca está associada a gravidade da doença e mau prognóstico para o paciente (MARTINELLI et al., 2016).

As alterações hemodinâmicas provocadas pela doença cardiovascular, afetam significativamente a hemodinâmica renal, o que leva a doença renal secundária em razão da disfunção cardíaca (ATKINS et al., 2009). As alterações hemodinâmicas, com baixo débito cardíaco, desencadeiam ativação neuro-hormonal através do SNS e do SRAA. Essas vias promovem vasoconstrição e a retenção de água e sódio, o que eleva a pressão venosa central e provoca aumento do volume sanguíneo e congestão de órgãos, consequentemente observa-se sobrecarga das câmaras, remodelamento e a progressão da doença cardíaca. A ativação neurohormonal tenta inicialmente preservar a TFG, porém provoca estresse renal, subclínico, ativo e contínuo que, subsequentemente, promove perda de massa e função renal. A congestão renal, por sua vez, também estimula o SNS e o SRAA, porém acaba aumentando a pressão do parênquima renal contribuindo para reduções progressivas da TFG e do fluxo de fluido tubular, induzindo a lesão renal ativa (ORVALHO; COWGILL, 2017).

O comprometimento da bomba cardíaca seja qual for a causa, resulta em diminuição do débito cardíaco e subsequente redução da pressão arterial sistêmica (PAS). Tais alterações promovem a estimulação de barorreceptores na parede arterial, que são ativados quando há redução do estiramento pelo baixo débito, 
enviando estímulo por via aferente até o centro vasomotor, que se encontra no bulbo encefálico. Ao processar a mensagem, o centro vasomotor envia resposta para aumentar o tônus simpático e a liberação de norepinefrina, que promove um estimulo de receptores $\alpha_{1}$ resultando em vasoconstrição arteriolar e aumento da resistência vascular periférica (PEREIRA et al., 2015).

O estimulo simpático também aumenta a frequência cardíaca de forma a compensar o baixo débito, através da ação nas células marcapasso do nó sinusal, aumentando a automaticidade das mesmas. Assim, com o aumento da frequência cardíaca há o aumento do débito cardíaco, que associado ao ajuste da resistência vascular periférica promoverá a manutenção da PAS. O aumento das catecolaminas circulantes contribuirá para o remodelamento cardíaco, resultando em hipertrofia e melhora da função miocárdica, consequentemente aumento do volume ejetado (PEREIRA et al., 2015).

A ativação compensatória do SNS ao longo do tempo passa a ser deletéria ao sistema cardiovascular, promovendo a progressão da doença cardíaca e alterações hemodinâmicas. $O$ aumento da frequência cardíaca diminui o tempo de enchimento diastólico, o que resulta em menor volume ejetado. $O$ aumento da resistência vascular periférica provoca maior resistência à via de saída ventricular, diminuindo o volume ejetado. As catecolaminas circulantes em excesso podem desenvolver arritmias e, cada vez mais, provocar o remodelamento miocárdico, piorando a insuficiência miocárdica. A ativação simpática, com o tempo, acaba por promover mais alterações hemodinâmicas, lesionando os sistemas cardiovascular e renal (PEREIRA et al., 2015).

A estimulação simpática também resulta em vários efeitos renais, como o aumento da reabsorção de sódio nos túbulos proximais e a secreção das células justaglomerulares. Portanto, níveis elevados de norepinefrina na circulação, estão correlacionados a um pior prognóstico em pacientes com insuficiência cardíaca (IC) (SCHRIER, 2006).

A diminuição da perfusão em órgãos alvos como os rins, cérebro e o coração, devido ao baixo débito cardíaco, desencadeia a ativação do SRAA. Os rins possuem células justaglomerulares que são sensíveis ao sódio e, quando há redução do sódio passando por essas células, há a liberação de renina produzida nessas células. A renina, através da circulação sanguínea, vai catalisar a transformação do angiotensinogênio, produzindo a angiotensina I, que possui pouco efeito vasoconstritor. Por meio da enzima conversora de angiotensina, a angiotensina I formará a angiotensina II (PEREIRA et al., 2015).

A angiotensina II é responsável por uma série de eventos, quando ativada. Como ação vascular, promove vasoconstrição para a manutenção da PAS, porém aumenta a pós carga que, em um coração insuficiente, aumenta a congestão e, no sistema renal, promove uma acentuada vasoconstrição nas arteríolas renais eferentes, aumentando a pressão de filtração glomerular. Como ação nervosa central, a angiotensina II atua no centro da sede, provocando aumento da ingestão hídrica e da volemia e, no sistema nervoso autônomo, promove o aumento de norepinefrina potencializando o efeito vasoconstritor (PEREIRA et al., 2015).

A liberação de vasopressina, um hormônio antidiurético, pela estimulação da angiotensina II, induz a absorção de água nos túbulos renais, aumentando o volume circulante, promovendo vasoconstrição e aumentando a pós carga (SCHRIER, 2006; PEREIRA et al., 2015). A angiotensina II como principal efeito estimula a secreção 
de aldosterona pelo córtex da adrenal. A aldosterona aumenta a reabsorção de sódio pelos túbulos renais que, consequentemente induz a reabsorção hídrica devido a alterações osmóticas, contribuindo para o aumento da volemia. A elevação prolongada da aldosterona induz a síntese de citocinas pro-inflamatórias, a fibrose e o aumento de matriz extracelular, o que exacerba a disfunção cardíaca com o remodelamento, o que, por sua vez, exacerba a SCR (PEREIRA et al., 2015).

A ativação dos mecanismos compensatórios neuro-hormonais promovendo o aumento da volemia e pós carga, bem como o remodelamento cardíaco e vascular, podem provocar colapso circulatório. A intensa ativação destes mecanismos, desenvolvem a suscetibilidade de pacientes com IC a lesões secundárias, como as alterações hemodinâmicas (PEREIRA et al., 2015).

$\mathrm{Na}$ doença renal progressiva, independente da natureza do insulto ao rim, observa-se a presença de estresse hemodinâmico e metabólico, desregulação metabólica e perda da integridade morfológica e funcional do epitélio tubular, levando à inflamação intersticial e fibrose epitelial. $O$ acúmulo de evidências na lesão renal aguda, propõe uma sequência de eventos adaptativos ou maladaptativos, podendo ser efetivos no reparo celular e que influenciam a prevenção ou então predispõe à lesão renal em curso de forma progressiva (POLZIN, 2011; HEZZELL et al., 2012; BONVENTRE, 2014; ZAGER, 2014; KELLUM; CHAWLA, 2016).

Destas linhas de evidência, a doença cardíaca, como exemplo, pode iniciar uma lesão aguda renal, comprometendo as células tubulares que, quando lesionadas, evoluem para necrose terminal ou apoptose, regeneram e reparam o dano celular, ou não conseguem se regenerar, mas sobrevivem à morte celular e mantém em um estado de pausa do ciclo celular (CHAWLA et al., 2014; ZUK; BONVENTRE, 2016). As células que são recuperadas do estado de apoptose, acabam participando da regulação das vias de sinalização mal-adaptativas com proliferação de miofibroblastos e fibrose intersticial, porém contribuem com a lesão renal em curso, mesmo na presença ou ausência do fator de estresse, como a disfunção cardíaca (FERENBACH; BONVENTRE, 2015; BASILE et al., 2016). Acredita-se que a disfunção cardíaca e seu manejo clínico levam a insultos renais isquêmicos e metabólicos, progredindo para uma lesão renal súbita e/ou progressiva (POLZIN, 2011; HEZZELL et al., 2012; CHAWLA et al., 2014).

\section{Diagnóstico}

Para classificar a SCR em cães, deve-se avaliar e caracterizar a presença e a gravidade da doença cardíaca e renal. Ambas devem ser avaliadas de forma independente, de acordo com a classificação para cada doença. O American College of Veterinary Internal Medicine (ACVIM), propõe a classificação da doença cardíaca em diferentes estágios (ATKINS et al., 2009).

O estágio A é composto por pacientes em alto risco de desenvolver doença cardíaca, mas que ainda não apresentam alterações estruturais, como os animais da raça Cavalier King Charles Spaniel, que apresentam predisposição a cardiopatia; o estágio B, identifica pacientes com alterações estruturais cardíacas (exemplo, o sopro), sem manifestações clínicas de insuficiência cardíaca. Este estágio é subdividido em B1 e B2, onde em B1 se enquadram pacientes assintomáticos sem evidências radiográficas ou ecocardiográficas de remodelamento cardíaco, e em B2 encontram-se pacientes assintomáticos que apresentam regurgitação valvar 
hemodinamicamente significativa com achados radiográficos ou ecocardiográficos de aumento das câmeras esquerdas; no estágio $C$, estão os pacientes com alterações estruturais e sinais clínicos de insuficiência cardíaca; e no estágio $D$, se enquadram os pacientes em estágio final de doença cardíaca com sinais clínicos de insuficiência cardíaca refratários à terapia padrão (ATKINS et al., 2009).

O International Renal Interest Society (IRIS), propôs a classificação e estadiamento da DRC e a graduação da insuficiência renal aguda (IRA) (IRIS, 2016), apesar do reconhecimento precoce da IRA ainda continua a ser um desafio, devido à falta de exames específicos para estadiar os graus iniciais (TAN et al., 2016; ELLIOTT; COWGILL, 2017).

O estágio 0 é representado por pacientes que possuem histórico com risco de desenvolver a DRC. São caracterizados por creatinina sérica $\leq 1,4 \mathrm{mg} / \mathrm{dL}$; no estágio 1, são observados pacientes não azotêmicos, porém com sinais de disfunção renal, como perda da capacidade de concentração urinária sem causa identificável, palpação renal anormal, alteração de imagem e estrutura renal, proteinúria de origem renal e resultados anormais de biopsia renal. A concentração de creatinina sérica também é $\leq 1,4 \mathrm{mg} / \mathrm{dL}$; no estágio 2 encontram-se pacientes com azotemia renal discreta e persistente. As manifestações clínicas de disfunção renal normalmente são leves ou ausentes e a creatinina sérica é de 1,4-2,0 mg/dL; o estágio 3, identifica pacientes com azotemia renal moderada e manifestações clínicas extrarrenais como poliúria, polidipsia e emagrecimento progressivo, 0 intervalo de creatinina sérica varia de 2,1-5,0mg/dL; e, no estágio 4, o paciente apresenta azotemia grave e manifestações clínicas sistêmicas de crises urêmicas, a creatinina sérica é $>5,0 \mathrm{mg} / \mathrm{DI}$ (IRIS, 2016).

Há ainda um subestadiamento quanto à perda urinária de proteína e a presença de hipertensão arterial sistêmica (HAS) de acordo com a IRIS. Pacientes que apresentam valores da relação proteína/creatinina urinária $(R P / C)<0,2$, são considerados não proteinúricos; $\mathrm{RP} / \mathrm{C}$ de 0,2-0,5 são suspeitos de apresentar proteinúria; e RP/C > 0.5, são pacientes proteinúricos. Já o subestadiamento, quanto à presença de HAS, é preconizado para a avaliação de risco de lesão em órgãos alvo (cérebro, olhos, coração e rins) secundariamente ao estado hipertensivo. Pacientes normotensos com mensuração da pressão arterial $<150 \mathrm{mmHg}$, possuem um risco mínimo de lesão em órgãos alvo; pacientes com 150-159mmHg, apresentam risco leve; já são considerados hipertensos, pacientes com a pressão arterial de 160-179mmHg e possuem um risco moderado de lesão em órgão alvo; e paciente com hipertensão grave $\geq 180 \mathrm{mmHg}$, tem alto risco de desenvolver lesões em outros órgãos (IRIS, 2016).

O estadiamento de DRC pela IRIS atualmente é baseada na concentração sérica de creatinina em jejum, porém sabe-se que as concentrações séricas de dimetilarginina simétrica (SDMA) são mais precoces para a identificação de disfunção renal. Com os resultados de novas pesquisas e informações sobre o uso de SDMA em pacientes veterinários, a IRIS lançou alguns critérios preliminares baseado em experiências do seu uso associado à quantificação de creatinina sérica. No estágio IRIS 1 com creatinina sérica $<1,4 \mathrm{mg} / \mathrm{dl}$, podem ser observados um aumento persistente de SDMA acima de $14 \mathrm{mg} / \mathrm{dl}$, demonstrando uma redução na função renal. Em doentes renais IRIS estágio 2 com escores de condição corporal baixa, a concentração sérica de SDMA $\geq 25 \mathrm{~g} / \mathrm{dl}$ indica que o grau de disfunção renal foi subestimado, neste caso é recomendado o tratamento para o estágio 3 da 
IRIS. O estágio IRIS 3 com escores de condição corporal baixa, SDMA $\geq 45 \mathrm{~g} / \mathrm{dl}$ pode indicar que foi subestimado o grau de disfunção renal, recomenda-se o tratamento para pacientes no estágio IRIS 4 (IRIS, 2016).

Mesmo sendo abordados de forma minuciosa, os protocolos de estadiamento para classificar doenças cardíacas e renais, com embasamento clínico e padrões atuais de diagnóstico, podem ser insensíveis para prever precocemente a síndrome cardiorrenal. Assim, o atraso da detecção da SCR e nas intervenções clínicas e terapêuticas do paciente podem influenciar no sucesso do tratamento (ORVALHO; COWGILL, 2017). Ressalta-se que as manifestações clínicas das doenças cardíacas e renais diferem entre as espécies, limitando assim a validade da obtenção de informações de dados humanos para aplicação veterinária, bem como comparações entre cães, gatos e outras espécies (POUCHELON et al., 2015).

O estadiamento clínico, avaliação e diagnóstico das doenças do eixo cardiovascular e renal, requerem a integração de diversas informações obtidas desde o início do atendimento com a anamnese, o histórico clínico e o exame físico, até exames complementares e testes diagnósticos convencionais. Exames de sangue e urina, medidas não invasivas para mensuração da pressão arterial, avaliação de imagens radiográficas e ultrassonográficas, são ferramentas de diagnóstico que estão disponíveis na rotina. O diagnóstico e estadiamento precisos e precoces são essenciais para a detecção de CvRD, e para o planejamento de planos terapêuticos e estabilização da doença (POUCHELON et al., 2015).

\section{Exames de imagem}

Exames de imagens são solicitados como método complementar para avaliação do paciente, sendo as modalidades de imagem radiográfica e ultrassonográfica, exames que fornecem expressões morfológicas da anatomia de diversos órgãos. A radiografia torácica é recomendada para estadiar doença cardíaca, mensurar o tamanho cardíaco (vertebral heart size - VHS), detectar alterações na silhueta cardíaca, avaliar estruturas vasculares e o padrão do parênquima pulmonar, determinando em última instância se há sinais de ICC. O exame de ecodopplercardiografia é recomendado para avaliar a morfologia e função cardíaca, se há a presença de lesões e alterações estruturais e para estimar os parâmetros hemodinâmicos relevantes (PEREIRA et al., 2015; POUCHELON et al., 2015). A imagem renal por meio da ultrassonografia abdominal é recomendada para alicerçar o diagnóstico e identificar alterações estruturais (RONCO; DI LULLO, 2014). Ainda, radiografias abdominais e ultrassonografia são recomendadas para auxiliar na detecção de anormalidades morfológicas em outros sítios e determinar a etiologia de doenças concomitantes. Juntos, essa combinação de dados de exames complementares, permite a detecção da presença, causa, gravidade e consequências das enfermidades que são observadas (POUCHELON et al., 2015).

\section{Exames laboratoriais convencionais}

Como ferramentas de diagnóstico, os ensaios bioquímicos convencionais e os biomarcadores são utilizados e aceitos para avaliação da função renal. A creatinina sérica, o SDMA e o clearance renal são usados para avaliar a TFG. A mensuração da albumina em soro e urina, as concentrações de proteína total e a $\mathrm{RP} / \mathrm{C}$ são usadas para avaliar a permesseletividade da membrana glomerular. As concentrações de glicose e aminoácidos na urina são usadas para avaliar a função 
tubular proximal, enquanto os eletrólitos séricos e as concentrações de bicarbonato refletem a capacidade do rim de manter o equilíbrio eletrolítico e ácido-base. A densidade urinária permite avaliar a capacidade de concentração renal (POUCHELON et al., 2015). A validade desses marcadores está bem estabelecida em casos de disfunção renal, embora sua especificidade para diferenciar lesão renal primária ou cardiovascular, ainda não tenha sido descrita (ORVALHO; COWGILL, 2017).

A creatinina sérica e o BUN vem sendo utilizado como marcadores clássicos para lesão renal, porém sabe-se que quando as concentrações desses marcadores estão elevadas no exame sanguíneo, o processo de lesão normalmente já está bem estabelecido, e muitas vezes é tarde demais para conseguir realizar intervenções terapêuticas para proteger os rins ou prevenir danos progressivos (POUCHELON et al., 2015; ORVALHO; COWGILL, 2017).

\section{Biomarcadores}

Existem biomarcadores no mercado com potencial para identificar doenças cardíacas e lesões renais de forma mais sensível que as avaliações clínicas convencionais. O uso pode facilitar o manejo terapêutico e acompanhamento clínico das doenças, com o intuito de prevenir o estabelecimento da síndrome cardiorrenal e melhorar o prognóstico do paciente (ORVALHO; COWGILL, 2017).

Os biomarcadores específicos para avaliação renal, que possuem potencial para sinalizar quando há estresse ou inadequada função celular, se localizam em epitélios tubulares renais funcionais ou outros sítios renais específicos. Pode-se observar lesão renal em curso ou progressiva, subsequente a disfunção cardíaca, antes que os métodos de diagnósticos convencionais sejam capazes de fazê-lo (ORVALHO; COWGILL, 2017).

De acordo com o estadiamento das doenças renais, no presente momento, a disfunção renal é avaliada por meio da mensuração de creatinina sérica ou estimativas da taxa de filtração glomerular (TFG), meios que são insensíveis ao diagnóstico precoce de lesão renal aguda. Acredita-se não haver um biomarcador singular e sim um painel de biomarcadores preditivos que detecta diferentes fases da lesão renal (indução, manutenção e reparo) e que deva auxiliar no suporte clínico do animal (ORVALHO; COWGILL, 2017).

Alguns dos marcadores sanguíneos como BUN, creatinina, cistatina $\mathrm{C}$ e SDMA são utilizados para a avaliação de filtração glomerular, dano glomerular e a proteinúria, e lesão tubular (ORVALHO; COWGILL, 2017). A descoberta e validação desses biomarcadores somados a outros novos biomarcadores como lipocalina associada a gelatinase de neutrófilos (NGAL), inosina sérica, clusterina sérica e urinária entre outros, poderão permitir o reconhecimento da lesão renal aguda (POUCHELON et al., 2015; ORVALHO; COWGILL, 2017) e a NGAL urinária já mostrou resultados promissores em cães (ORVALHO; COWGILL, 2017).

Os resultados dos estudos das proteínas urinárias como biomarcadores são promissores e refletem funções ou processos celulares específicos para os rins, que são interrompidos por eventos fisiopatológicos secundários à isquemia, lesão ou estresse celular. Proteína ligadas ao retinol, cistatina $C$, cistatina $B$, molécula de lesão renal-1 (RIM-1) (ALGE; ARTHUR, 2015; YERRAMILLI et al., 2016), NGAL (JUNG et al., 2018), interleucina-18, proteína ligadora de ácido graxo hepático (LFABP) (ALGE; ARTHUR, 2015; KASHANI; KELLUM, 2015; YERRAMILLI et al., 
2016; ZUK; BONVENTRE, 2016), inibidor de metaloproteinase-2 e proteína de ligação do fator de crescimento semelhante à insulina-7 estão entre as mais estudadas atualmente (ALGE; ARTHUR, 2015; YERRAMILLI et al., 2016).

Com o advento do SDMA, há uma nova avaliação diagnóstica que prevê o início da DRC mais precocemente do que a creatinina sérica, porém ainda é um teste de função que pode não discriminar a lesão renal subclínica (BROWN, 2015; RELFORD et al., 2016). Tanto a creatinina sérica quanto o SDMA são marcadores de função renal que podem apresentar falhas em detectar a lesão inicial e ativa, secundária a doença cardíaca e seu manejo clínico, principalmente quando compensada pela adaptação da massa renal residual. Somente haverá envolvimento renal, observado por meio de avaliação funcional, quando a lesão ativa ultrapassar o reparo fisiológico, o que torna evidente a disfunção do órgão (RELFORD et al., 2016; ORVALHO; COWGILL, 2017).

O SDMA, é um produto do metabolismo de proteínas intranucleares, filtradas livremente pelos rins e os seus níveis séricos correlacionam inversamente com as medidas de TFG em pessoas, ratos, camundongos, cães e gatos, sugerindo-a como um bom marcador de doença renal (MARESCAU et al., 1997). Estudos longitudinais em cães e gatos com DRC mostraram que, o nível de SDMA aumentou meses antes da creatinina sérica quando houve, em média, uma redução de $40 \%$ na TFG (RELFORD et al., 2016). Os valores de SDMA aumentam em concordância com a diminuição da taxa de filtração glomerular, identificando melhor a perda da função renal (HALL et al., 2014). Existem evidências que apoiam a SDMA como um biomarcador renal endógeno específico, não influenciado por fatores extrarrenais, como massa corpórea, nutrição e doenças concomitantes. Com o seu uso, os veterinários têm a oportunidade potencial de diagnosticar precocemente a doença renal em cães, sendo duas vezes mais sensível em comparação com o uso de creatinina sérica (RELFORD et al., 2016).

A inosina sérica, a clusterina urinária e a cistatina urinária, são biomarcadores que possuem origem exclusiva nos epitélios tubulares renais, associado ao estresse celular, e sua avaliação analítica é altamente específica (YERRAMILLI et al., 2016). Apresentam a sensibilidade e especificidade em potencial para indicar precocemente o envolvimento renal, subsequente à disfunção cardíaca em comparação aos diagnósticos convencionais de lesão renal (HEUNG; CHAWLA, 2014; ALGE; ARTHUR, 2015) .

A proteína NGAL foi identificada com aumento de mais de 10 vezes nos epitélios tubulares renais nas primeiras horas após lesões renais isquêmicas, obstrutivas e tóxicas em pacientes humanos com lesão renal aguda (HEUNG; CHAWLA, 2014; ALGE; ARTHUR, 2015). Porém, já foi identificado em vários epitélios e não possui especificidade única para a lesão renal, podendo ser influenciada por comorbidades (ORVALHO; COWGILL, 2017). A avaliação com NGAL identifica lesão tubular que precede a perda funcional renal em vários dias e o aumento da creatinina sérica sugere perda subsequente da função excretora renal (HAASE et al., 2013). É provável que os túbulos renais possam ser lesionados primariamente, por serem mais suscetíveis à diminuição da perfusão e ao dano hipóxico, provocado pela insuficiência cardíaca, em relação ao córtex renal que, em um momento inicial permanece intacto, mantendo a filtração glomerular (VAN DEURSEN et al., 2014). 
O uso dos biomarcadores cardíacos específicos combinados com os novos biomarcadores de lesão renal podem fornecer melhor compreensão da síndrome cardiorrenal em cães (ORVALHO; COWGILL, 2017). Muitos dos novos marcadores estão sendo avaliados e validados em animais (HOKAMP; NABITY, 2016).

Em detrimento dos avanços, os biomarcadores cardíacos como a porção terminal do peptídeo natriurético do tipo B (NT-proBNP) e a troponina I cardíaca (cTnl) são os biomarcadores mais utilizados para doença cardiovascular. O NTproBNP é liberado pelo miocárdio, sendo capaz de detectar o estresse de miócitos, em resposta a sobrecarga de volume ou de pressão, revelando congestão ao nível celular. Sua principal função é regular o volume do plasma promovendo a natriurese. A troponina I cardíaca é um indicador bem estabelecido de doença ou lesão cardíaca, sendo um marcador de lesão dos miócitos, liberada quando há lesão e desintegração dos sarcômeros cardíacos (HEZZELL et al., 2012). Contudo, NTproBNP e a cTnl são parcialmente excretados pelos rins, sendo os seus valores dependentes da função renal, o que deve ser lembrado ao avaliar pacientes com disfunção cardíaca e renal. A interpretação combinada desses biomarcadores no contexto de uma condição clínica, podem auxiliar na conduta terapêutica (ORVALHO; COWGILL, 2017).

\section{Tratamento}

O manejo terapêutico para paciente com SCR é desafiador. Doentes renais dependem do manejo com fluidoterapia, em principal atenção aos distúrbios hidroeletrolíticos, e também necessitam de precaução quanto à quantidade e qualidade da ingestão de proteína e fósforo (POLZIN, 2011). Já os cardiopatas com IC necessitam de diurese em casos de sinais de congestão venosa, e recebem suplementação protéica, com proteínas de alta qualidade devido à caquexia cardíaca (ATKINS et al., 2009).

A diurese faz parte do tratamento para animais com ICC, pois a congestão vascular não causa apenas os sinais clínicos da ICC, como também 0 remodelamento cardíaco e a progressão da doença cardíaca. Entretanto, a diurese excessiva pode agredir ainda mais a função renal. Os diuréticos de alça podem reduzir a congestão renal, melhorar a TFG e retardar a progressão da DRC, porém em doses excessivas, eles podem diminuir a perfusão renal e, consequentemente, a TFG (METRA et al., 2012). O paciente em tratamento com diurético deve ser avaliado e, em casos de discreto a moderado aumento da creatinina sérica, o que deve ser esperado, pode ser tolerado dentro dos limites e com acompanhamento clínico (POUCHELON et al., 2015; ORVALHO; COWGILL, 2017). Em casos de azotemia durante o tratamento de ICC aguda, deve-se realizar a redução da dose diária total (dosagem e/ou frequência de administração) de diuréticos e, para melhores resultados, associar o uso de vasodilatadores, venosos ou arteriais, com intuito de reduzir a pré carga e pós carga (ATKINS et al., 2009; POUCHELON et al., 2015).

A combinação de diuréticos deve ser usada com cautela em pacientes com síndrome cardiorrenal, pois os diuréticos de alça associados aos diuréticos tiazídicos tem efeito sinérgico, que pode causar depleção excessiva de volume e distúrbios eletrolíticos, devendo ser monitorados nestes pacientes (ORVALHO; COWGILL, 2017). 
O foco do tratamento de pacientes com ICC é diminuir a congestão vascular, na tentativa de diminuir o volume intravascular e as pressões de enchimento cardíaco, para que os sinais clínicos desapareçam. Uma abordagem para estes pacientes é diminuir a resistência vascular sistêmica com a vasodilatação, que diminui as pressões de enchimento cardíaco e aumenta o fluxo da via de saída e, consequentemente, o débito cardíaco. Vasodilatadores como o nitroprussiato de sódio e a nitroglicerina são os principais fármacos utilizados em pacientes com ICC emergenciais. No entanto, o uso indevido e não controlado também pode causar hipotensão com consequente diminuição da pressão e perfusão renal, provocando lesão renal (ROSARIO et al., 1998).

Inibidores da enzima conversora de angiotensina (iECA) são benéficos em doenças cardiovasculares e renais, pois promovem a melhora das manifestações clínicas. Os iECAs bloqueiam a conversão de angiotensina I em angiotensina II, causando uma redução na resistência arteriolar sistêmica e um grau moderado de vasodilatação. Outras ações que são prevenidas com o uso de iECA são o aumento da volemia, com a reabsorção de sódio e subsequente absorção de água, a fibrose miocárdica e o remodelamento cardíaco. Em casos de hipotensão, deve-se evitar o uso de iECAs, pois promovem vasodilatação arteriolar eferente renal o que pode afetar a TFG (ATKINS et al., 2009). Antagonistas dos receptores mineralocorticóides (bloqueadores da aldosterona) têm potencial para proteção renal e cardíaca, portanto, o uso de espironolactona nesses pacientes pode ser benéfico, mas ainda necessita de mais estudos (ATKINS et al., 2009; BERNAY et al., 2010).

Em uma análise com pacientes humanos, foi identificado que a inibição do SRAA foi benéfica em pacientes com disfunção renal. Portanto, manter esses medicamentos em pacientes com SCR, podem ser favoráveis, apesar de ser observado um leve aumento nos marcadores da função renal (CLARK et al., 2015).

Inotrópicos positivos são indicados em pacientes hemodinamicamente instáveis, apresentando baixo débito cardíaco e baixa pressão arterial sistólica. 0 pimobendan melhora a função sistólica e possui ação vasodilatadora, o que pode aumentar a TFG (LANTIS et al., 2011). Em casos específicos, pode-se optar pela infusão intravenosa de dobutamina para aumentar o débito cardíaco e melhorar a perfusão renal (POUCHELON et al., 2015). A digoxina e outros fármacos com excreção renal predominante, exigem monitoração e acompanhamentos mais próximos, pois podem ser tóxicos e prejudiciais tornando necessária a redução ou até mesmo suspensão (ORVALHO; COWGILL, 2017).

Os ácidos graxos ômega-3 são um suplemento oral, extraído de óleos de peixes recomendado como antioxidante, estimulante do apetite (POLZIN, 2011), antiarrítmicos, anti-inflamatórios e anti-ateroescleróticos. Além de, auxiliar na redução de triglicerídeos e promoverem melhora nas alterações hemodinâmicas como a pressão arterial, enchimento diastólico do ventrículo esquerdo, frequência cardíaca e função endotelial (ADKINS; KELLEY, 2010).

O tratamento das disfunções renais, como a IRA ou DRC, envolve uma combinação de fluidoterapia, para manutenção de volume e eletrólitos, no intuito de permitir uma perfusão renal suficiente, evitando a sobrecarga de fluidos e o desequilíbrio eletrolítico, em associação com fármacos para tratamento suporte. São comuns em pacientes nefropatas o uso de diuréticos para tratamento de sobrecarga de volume e hipercalemia, agentes antihipertensivos, iECA, protetores 
gastrintestinais, agentes alcalinizantes, agentes estimuladores da eritropoiese, ligantes de fosfato e ajuste dietético, com baixa proteína (DEFRANCESCO, 2008).

Contudo, sabe-se que tanto o coração quanto o rim são afetados por alterações no volume de fluidos intravasculares, por alterações na pressão sanguínea sistêmica, seja por hipertensão ou hipotensão, e por tratamentos comumente empregados em casos de CvRD, como diuréticos, vasodilatadores, antiarrítmicos, inotrópicos positivos, fluidos suplementares e reposições eletrolíticas, usados para corrigir esses desequilíbrios (ATKINS et al., 2009; POUCHELON et al., 2015). Conseguir um equilíbrio nesses pacientes se torna uma atividade mais difícil quando o coração e os rins são concomitantemente disfuncionais (POUCHELON et al., 2015).

O uso dos novos biomarcadores renais pode auxiliar a instituição de protocolos de tratamento mais agressivos, eficientes e, ao mesmo tempo, seguros para os pacientes. Pacientes que apresentam disfunção renal normalmente recebem um tratamento abaixo do ideal para as condições cardiovasculares concomitantes, mesmo que possam se beneficiar das terapias padrões, o que contribui para o agravamento do prognóstico em pacientes com síndrome cardiorrenal (ORVALHO; COWGILL, 2017).

\section{CONSIDERAÇÕES FINAIS}

O conhecimento, capacidade de identificação, definição, compreensão da fisiopatogenia e da complexidade dos cuidados necessários na SCR, são primordiais para oferecer o melhor acompanhamento e manejo terapêutico a esses pacientes. A SCR exige uma abordagem multidisciplinar, combinando as experiências de especialistas em cardiologia e nefrologia.

É sublinhada a necessidade de elaborar recomendações para a prevenção e estratégias terapêuticas eficazes para a SCR, pois falta consenso quanto aos possíveis fármacos disponíveis no mercado que podem ser úteis no seu manejo. Um conhecimento mais aprofundado acerca dos mecanismos neuro-hormonais envolvidos na SCR poderá conferir potenciais alvos de intervenção terapêutica desses pacientes.

Há maior necessidade de estudos com biomarcadores capazes de detectar estágios iniciais da SCR. Assim, a busca de exames com potencial para identificar interações cardiorrenais, que podem ser subclínicas, modifica as decisões terapêuticas no tratamento das doenças cardíacas com intuito da preservação do envolvimento renal. Manejos terapêuticos, precisos e precoces, podem prevenir o agravamento da doença cardíaca e da lesão renal mútua, aumentando as chances de sobrevida do paciente assim como a qualidade de vida do mesmo.

O conhecimento entre os médicos veterinários sobre as diferentes ferramentas de diagnóstico é indispensável, de modo a detectar precocemente a SCR, e consequentemente instituir a terapia adequada.

\section{REFERÊNCIAS}

ADKINS, Y.; KELLEY, D. Mechanisms underlying the cardioprotective effects of omega-3 polyunsaturated fatty acids. Journal of Nutritional Biochemistry, v. 21, n. 9, p. 781-792, 2010. DOI: 10.1016/j.jnutbio.2009.12.004. Disponivel em: https://www.ncbi.nlm.nih.gov/pubmed/20382009. 
ALGE, J. L.; ARTHUR, J. M. Biomarkers of AKI: a review of mechanistic relevance and potential therapeutic implications. Clinical Journal of the American Society of Nephrology, v. 10, n. 1, p. 147-55, Jan 7 2015. ISSN 1555-9041. DOI: 10.2215/CJN.12191213.

em: https://www.ncbi.nlm.nih.gov/pubmed/25092601.

ATKINS, C.; BONAGURA, J.; ETTINGER, S.; FOX, P.; GORDON, S.; HAGGSTROM, J.; HAMLIN, R.; KEENE, B.; LUIS-FUENTES, V.; STEPIEN, R. Guidelines for the diagnosis and treatment of canine chronic valvular heart disease. Journal of Veterinary Internal Medicine, v. 23, n. 6, p. 1142-50, Nov-Dec 2009. ISSN 0891-6640. DOI: 10.1111/j.1939-1676.2009.0392.x. Disponível em: < https://www.ncbi.nlm.nih.gov/pubmed/19780929 >.

BAGSHAW, S. M.; HOSTE, E. A.; BRAAM, B.; BRIGUORI, C.; KELLUM, J. A.; MCCULLOUGH, P. A.; RONCO, C. Cardiorenal Syndrome Type 3. Pathophysiologic and Epidemiologic Considerations, v. 182, p. 137-157, 2018. DOI: $\quad 10.1159 / 000349971 . \quad$ Disponível em: < https://www.ncbi.nlm.nih.gov/pubmed/23689660.

BASILE, D. P.; BONVENTRE, J. V.; MEHTA, R.; NANGAKU, M.; UNWIN, R.; ROSNER, M. H.; KELLUM, J. A.; RONCO, C.; ADQI XIII WORK GROUP. Progression after $\mathrm{AKI}$ : understanding maladaptive repair processes to predict and identify therapeutic treatments. Journal of the American Society of Nephrology, v. 27, n. 3, p. 687-97, 2016. DOI: 10.1681/ASN.2015030309. Disponivel em: https://www.ncbi.nlm.nih.gov/pubmed/26519085.

BERNAY, F.; BLAND, J. M.; HÄGGSTRÖM, J.; BADUEL, L.; COMBES, B.; LOPEZ, A.; KALTSATOS, V. Efficacy of spironolactone on survival in dogs with naturally occurring mitral regurgitation caused by myxomatous mitral valve disease. Journal of Veterinary Internal Medicine, v. 24, n. 2, p. 331-41, Mar-Apr 2010. ISSN 0891 6640. Disponível em: < http://dx.doi.org/10.1111/j.1939-1676.2009.0467.x.

BONVENTRE, J. V. Primary proximal tubule injury leads to epithelial cell cycle arrest, fibrosis, vascular rarefaction, and glomerulosclerosis. Kidney International Supplements (2011), n.4, v.1, p. 39-44, Oct 2014. Doi: 10.1038/kisup.2014.8. Disponivel em: https://www.ncbi.nlm.nih.gov/pmc/articles/PMC4536970/.f

BROWN, S. A. Symmetricdimethylarginine (SDMA): new biomarkerof renal function in catsanddogs (2015). International Renal Interest Society, University of Georgia, Athens, USA. Disponível em: http://iris-kidney.com/pdf/symmetricdimethylarginine.pdf. Acesso em: 04 set. 2018.

CHAWLA, L. S.; EGGERS, P. W.; STAR, R. A.; KIMMEL, P. L. Acute kidney injury and chronic kidney disease as interconnected syndromes. The New England Journal of Medicine, v. 371, p. 58-66, 2014. DOI: 10.1056/NEJMra1214243. Disponivel em: < https://www.ncbi.nlm.nih.gov/pubmed/24988558. 
CLARK, H.; KRUM, H.; HOPPER, I. Worsening renal function during reninangiotensinaldosterone system inhibitor initiation and long-term outcomes in patients with left ventricular systolic dysfunction. European Journal of Heart Failure, v. 16, p. 41-8, 2015. DOI: 10.1002/ejhf.13. Disponivel em: https://www.ncbi.nlm.nih.gov/pubmed/24453097.

COWGILL, L. D.; POLZIN, D. J.; ELLIOTT, J.; NABITY, M. B.; SEGEV, G.; GRAUER, G. F.; BROWN, S.; LANGSTON, C.; VAN DONGEN, A. M. Is progressive chronic kidney disease a slow acute kidney injury? Veterinary Clinics of North America: Small Animal Practice, v. 46, n. 6, p. 995-1013, 2016. DOI: 10.1016/j.cvsm.2016.06.001. https://www.ncbi.nlm.nih.gov/pubmed/27593574.

Disponível em:

DEFRANCESCO, T. C. Maintaining fluid and electrolyte balance in heart failure. Veterinary Clinics of North America: Small Animal Practice, v. 38, n. 3, p. 72745, xiv, May 2008. ISSN 0195-5616. Disponível em: < http://dx.doi.org/10.1016/j.cvsm.2008.02.005 >.

ELLIOTT, J.; COWGILL, L. Diagnostic algorithms for grading of acute kidney and stagingthe chronic kidney disease patient. In: ELLIOTT, J. e GRAUER, G. (Ed.). BSAVA manual of canine and feline nephrology and urology. 3. Gloucester: British small animal veterinary association 2017.

FERENBACH, D. A.; BONVENTRE, J. V. Mechanisms of maladaptive repair after AKI leading to accelerated kidney ageing and CKD. Nature Reviews Nephrology, v. 11, p. 264-76, 2015. DOI: 10.1038/nrneph.2015.3. Disponivel em: https://www.ncbi.nlm.nih.gov/pubmed/25643664.

FREITAS, R. A.; SILVA, B. R. S. A.; ATHAR, C. A. A.; MARINHO, J. P. M.; VEIGA, C. C. P.; PAIVA, J. P. Estadiamento da doença renal crônica em cães domésticos com doença valvar crônica. Revista Brasileira de Medicina Veterinária, v. 38, n. 2, p. 31-39, 2016.

HAASE, M.; MÜLLER, C.; DAMMAN, K.; MURRAY, P. T.; KELLUM, J. A.; RONCO, C.; MCCULLOUGH, P. A. Pathogenesis of cardiorenal syndrome type 1 in acute decompensated heart failure: workgroup statements from the eleventh consensus conference of the Acute Dialysis Quality Initiative (ADQI). Contributions to nephrology, v. 182, p. 99-116, 2013. ISSN 0302-5144. Disponível em: < http://dx.doi.org/10.1159/000349969 >.

HALL, J. A.; YERRAMILLI, M.; OBARE, E.; YERRAMILLI, M.; JEWELL, D. E. Comparison of serum concentrations of symmetric dimethylarginine and creatinine as kidney function biomarkers in cats with chronic kidney disease. Journal of Veterinary Internal Medicine, v. 28, n. 6, p. 1676-83, Nov-Dec 2014. ISSN 08916640. Disponível em: < http://dx.doi.org/10.1111/jvim.12445 >. 
HEUNG, M.; CHAWLA, L. S. Acute kidney injury: gateway to chronic kidney disease. Nephron Clinical Practice, v. 127, n. 1-4, p. 30-4, 2014. ISSN 1660-2110. Disponível em: < http://dx.doi.org/10.1159/000363675 >.

HEZZELL, M. J.; BOSWOOD, A.; CHANG, Y. M.; MOONARMART, W.; SOUTTAR, K.; ELLIOTT, J. The combined prognostic potential of serum high-sensitivity cardiac troponin I and N-terminal pro-B-type natriuretic peptide concentrations in dogs with degenerative mitral valve disease. Journal of Veterinary Internal Medicine, v. 26, n. 2, p. 302-11, Mar-Apr 2012. ISSN 0891-6640. Disponível em: < http://dx.doi.org/10.1111/j.1939-1676.2012.00894.x >.

HOKAMP, J. A.; NABITY, M. B. Renal biomarkers in domestic species. Veterinary Clinical Pathology, n.45, v.1, p.28-56, Mar 2016. DOI: 10.1111/vcp.12333. Disponivel em: https://www.ncbi.nlm.nih.gov/pubmed/26918420.

IRIS, International Renal Interest Society. GUIDELINES. http://www.iriskidney.com/guidelines/index.html, 2016. Acesso em: 01 de setembro de 2018.

JESSUP, M.; COSTANZO, M. R. The cardiorenal syndrome: do we need a change of strategy or a change of tactics? Journal of the American College of Cardiology. United States, v.53, 2009. p.597-9. ISBN 1558-3597. DOI: 10.1016/j.jacc.2008.11.012. Disponivel em: https://www.ncbi.nlm.nih.gov/pubmed/19215834.

JUNG, H. B.; KANG, M. H.; PARK, H. M. Evaluation of serum neutrophil gelatinaseassociated lipocalin as a novel biomarker of cardiorenal syndrome in dogs. The Journal of Veterinary Diagnostic Investigation, v. 30, n. 3, p. 386-391, May 2018. Doi: $\quad 10.1177 / 1040638718758430 . \quad$ Disponivel em: https://www.ncbi.nlm.nih.gov/pubmed/29429401.

KANG, J. G.; YU, M. Y.; LEE, H.; KIM, D. K.; JOO, K. W.; KIM, Y. S.; YANG, S. H. Blood pressure management and progression of chronic kidney disease in a canine remnant kidney model. General Physiology and Biophysics, v.37, n.3, p.243-252, May 2018. Doi: 10.4149/gpb_2017055. Disponivel em: https://europepmc.org/abstract/med/29589837.

KASHANI, K.; KELLUM, J. A. Novel biomarkers indicating repair or progression after acute kidney injury. Current Opinion in Nephrology and Hypertension, n.24, v.1, p. 21-7, 2015. DOI: 10.1097/MNH.0000000000000090. Disponivel em: https://www.ncbi.nlm.nih.gov/pubmed/25415614.

KELLER, S. P.; KOVACEVIC, A.; HOWARD, J.; SCHWEIGHAUSER, A.; FRANCEY, $\mathrm{T}$. Evidence of cardiac injury and arrhythmias in dogs with acute kidney injury. Journal of Small Animal Practice, v. 57, n. 8, p. 402-8, Aug 2016. DOI: 10.1111/jsap.12495. Disponivel em: https://www.ncbi.nlm.nih.gov/pubmed/27357259.

KELLUM, J. A.; CHAWLA, L. S. Cell-cycle arrest and acute kidney injury: the light and the dark sides. Nephrology Dialysis Transplantation, v.31, n.1, p.16-22, Jan 
2016.

DOI:

$10.1093 /$ ndt/gfv130.

Disponivel

em:

https://www.ncbi.nlm.nih.gov/pubmed/26044835.

LANGHORN, R.; OYAMA, M. A.; KING, L. G.; MACHEN, M. C.; TRAFNY, D. J.; THAWLEY, V.; WILLESEN, J. L.; TARNOW, I.; KJELGAARD-HANSEN, M. Prognostic importance of myocardial injury in critically ill dogs with systemic inflammation. Journal of Veterinary Internal Medicine, v. 27, n. 4, p. 895-903, JulAug 2013. ISSN 0891-6640. Disponível em: < http://dx.doi.org/10.1111/jvim.12105 >. LANTIS, A. C.; ATKINS, C. E.; DEFRANCESCO, T. C.; KEENE, B. W.; WERE, S. R. Effects of furosemide and the combination of furosemide and the labeled dosage of pimobendan on the circulating renin-angiotensin-aldosterone system in clinically normal dogs. American Journal of Veterinary Research, v. 72, n. 12, p. 1646-51, Dec 2011. ISSN 0002-9645. Disponível em: < http://dx.doi.org/10.2460/ajvr.72.12.1646 >.

LIANG, K. V.; WILLIAMS, A. W.; GREENE, E. L.; REDFIELD, M. M. Acute decompensated heart failure and the cardiorenal syndrome. Critical Care Medicine, v. 36, n. 1 Suppl, p. S75-88, Jan 2008. ISSN 1530-0293. DOI: 10.1097/01.CCM.0000296270.41256.5C. Disponível em: < https://www.ncbi.nlm.nih.gov/pubmed/18158481 >.

MARESCAU, B.; NAGELS, G.; POSSEMIERS, I.; BROE, M. E.; BECAUSE, I.; BILLIOUW, J. M.; LORNOY, W.; DEYN, P. P. Guanidino compounds in serum and urine of nondialyzed patients with chronic renal insufficiency. Metabolism, v. 46, n. 9, p. 1024-31, Sep 1997. ISSN 0026-0495. Disponível em: < https://www.ncbi.nlm.nih.gov/pubmed/9284891.

MARTINELLI, E.; LOCATELLI, C.; BASSIS, S.; CROSARA, S.; PALTRINIERI, S.; SCARPA, P.; SPALLA, I.; ZANABONI, A. M.; QUINTAVALLA, C.; BRAMBILLA, P. Preliminary Investigation of Cardiovascular-Renal Disorders in Dogs with Chronic Mitral Valve Disease. Journal of Veterinary Internal Medicine, v. 30, n. 5, p. 16121618, Sep 2016. ISSN 1939-1676. DOI: 10.1111/jvim.14524. Disponível em: < https://www.ncbi.nlm.nih.gov/pubmed/27717188 >.

METRA, M.; DAVISON, B.; BETTARI, L.; SUN, H.; EDWARDS, C.; LAZZARINI, V.; PIOVANELLI, B.; CARUBELLI, V.; BUGATTI, S.; LOMBARDI, C.; COTTER, G.; DEI $\mathrm{CAS}$, L. Is worsening renal function an ominous prognostic sign in patients with acute heart failure? The role of congestion and its interaction with renal function. Circulation: Heart Failure, v. 5, n. 1, p. 54-62, Jan 2012. ISSN 1941-3289. Disponível em: < http://dx.doi.org/10.1161/circheartfailure.111.963413 >.

O'NEILL, D. G.; ELLIOTT, J.; CHURCH, D. B.; MCGREEVY, P. D.; THOMSON, P. C.; BRODBELT, D. C. Chronic kidney disease in dogs in UK veterinary practices: prevalence, risk factors, and survival. Journal of Veterinary Internal Medicine, v. 27, n. 4, p. 814-21, Jul-Aug 2013. ISSN 0891-6640. Disponível em: < http://dx.doi.org/10.1111/jvim.12090 >. 
ORVALHO, J. S.; COWGILL, L. D. Cardiorenal Syndrome: Diagnosis and Management. Veterinary Clinics of North America: Small Animal Practice, v. 47, n. 5, p. 1083-1102, Sep 2017. ISSN 1878-1306. DOI: 10.1016/j.cvsm.2017.05.004. Disponível em: < https://www.ncbi.nlm.nih.gov/pubmed/28669432 >.

PEREIRA, G.; YAMATO, R.; LARSSON, M. Insuficiência Cardíaca Congestiva. In: JERICÓ, M.; ANDRADE NETO, J., et al (Ed.). Tratado de medicina interna de cães e gatos. 1. Rio de Janeiro: Roca, v.1, 2015. p.1099-1118.

POLZIN, D. J. Chronic kidney disease in small animals. Veterinary Clinics of North America: Small Animal Practice, v. 41, n. 1, p. 15-30, Jan 2011. ISSN 0195-5616. Disponível em: < http://dx.doi.org/10.1016/j.cvsm.2010.09.004 >.

POUCHELON, J. L.; POUCHELON, J. L.; ATKINS, C. E.; BUSSADORI, C.; OYAMA, M. A.; et al. Cardiovascular-renal axis disorders in the domestic dog and cat: a veterinary consensus statement. Journal of Small Animal Practice, v. 56, n. 9, p. 537-52, Sep 2015. ISSN 1748-5827. DOI: 10.1111/jsap.12387. Disponível em: < https://www.ncbi.nlm.nih.gov/pubmed/26331869 >.

RANGASWAMI, J.; BHALLA, V.; BLAIR, J. E. A.; CHANG, T. I.; COSTA, S.; et al. Cardiorenal Syndrome: Classification, Pathophysiology, Diagnosis, and Treatment Strategies: A Scientific Statement From the American Heart Association. Circulation, v. 139, p. 00-00, 2019. DOI: 10.1161/CIR.0000000000000664. Disponivel em: https://www.ncbi.nlm.nih.gov/pubmed/30852913.

RELFORD, R.; ROBERTSON, J.; CLEMENTS, C. Symmetric Dimethylarginine: Improving the Diagnosis and Staging of Chronic Kidney Disease in Small Animals. Veterinary Clinics of North America: Small Animal Practice, v. 46, n. 6, p. 94160, Nov 2016. ISSN 0195-5616. Disponível em: < http://dx.doi.org/10.1016/j.cvsm.2016.06.010 >.

RONCO, C.; DI LULLO, L. Cardiorenal syndrome. Heart Failure Clinics, v. 10, n. 2, p. 251-80, Apr 2014. ISSN 1551-7136. DOI: 10.1016/j.hfc.2013.12.003. Disponível em: < https://www.ncbi.nlm.nih.gov/pubmed/24656104 >.

RONCO, C.; HAAPIO, M.; HOUSE, A. A.; ANAVEKAR, N.; BELLOMO, R. Cardiorenal syndrome. Journal of the American College of Cardiology, v. 52, n. 19, p. 1527-39, Nov 4 2008. ISSN 0735-1097. Disponível em: < http://dx.doi.org/10.1016/j.jacc.2008.07.051 >.

RONCO, C.; MCCULLOUGH, P.; ANKER, S. D.; ANAND, I.; ASPROMONTE, N.; et al. Cardio-renal syndromes: report from the consensus conference of the acute dialysis quality initiative. European Heart Journal, v. 31, n. 6, p. 703-11, Mar 2010. ISSN 1522-9645. DOI: 10.1093/eurheartj/ehp507. Disponível em: < https://www.ncbi.nlm.nih.gov/pubmed/20037146 >.

ROSARIO, L. B.; STEVENSON, L. W.; SOLOMON, S. D.; LEE, R. T.; REIMOLD, S. $C$. The mechanism of decrease in dynamic mitral regurgitation during heart failure 
treatment: importance of reduction in the regurgitant orifice size. Journal of the American College of Cardiology, v. 32, n. 7, p. 1819-1824, $1998 . \quad$ Disponivel em: https://www.ncbi.nlm.nih.gov/pubmed/9857857.

SCHRIER, R. W. Role of diminished renal function in cardiovascular mortality: marker or pathogenetic factor? Journal of the American College of Cardiology, v. 47, n. 1, p. 1-8, Jan 3 2006. ISSN 0735-1097. Disponível em: < http://dx.doi.org/10.1016/j.jacc.2005.07.067 >.

SUZUKI, H.; MATSUMOTO, A.; MURAKAMI, M.; NAITOH, M.; SARUTA, T. Canine Model of Cardiorenal Failure | OMICS International. Journal of Nephrology \& Therapeutics, v. 2, n. 6, p. 1, 2012. ISSN 2161-0959. Disponível em: < https://www.omicsonline.org/canine-model-of-cardiorenal-failure-2161-

0959.1000127. php?aid=9222\&view=mobile $>$.

TAN, H. L.; YAP, J. Q.; QIAN, Q. Acute kidney injury: tubular markers and risk for chronic kidney disease and end-stage kidney failure. Blood Purification, n.41; v. 13, p.144-50, 2016. DOI: 10.1159/000441269. Disponivel em: https://www.ncbi.nlm.nih.gov/pubmed/26764483.

VAN DEURSEN, V. M.; DAMMAN, K.; VOORS, A. A.; VAN DER WAL, M. H.; JAARSMA, T.; VAN VELDHUISEN, D. J.; HILLEGE, H. L. Prognostic value of plasma neutrophil gelatinase-associated lipocalin for mortality in patients with heart failure. Circulation: Heart Failure, v. 7, n. 1, p. 35-42, Jan 2014. ISSN 1941-3289. Disponível em: < http://dx.doi.org/10.1161/circheartfailure.113.000242 >.

WALDUM, B.; OS, I. The cardiorenal syndrome: what the cardiologist needs to know. Cardiology, v. 126, n. 3, p. 175-86, 2013. ISSN 0008-6312. Disponível em: < http://dx.doi.org/10.1159/000353261 >.

WEHNER, A.; HARTMANN, K.; HIRSCHBERGER, J. Associations between proteinuria, systemic hypertension and glomerular filtration rate in dogs with renal and non-renal diseases. Veterinary Record, v. 162, n. 5, p. 141-7, Feb 22008. ISSN 0042-4900. Disponível em: https://www.ncbi.nlm.nih.gov/pubmed/18245745.

YERRAMILLI, M.; FARACE, G.; QUINN, J. Kidney Disease and the Nexus of Chronic Kidney Disease and Acute Kidney Injury: The Role of Novel Biomarkers as Early and Accurate Diagnostics. Veterinary Clinics of North America: Small Animal Practice, v. 46, n. 6, p. 961-93, Nov 2016. ISSN 0195-5616. Disponível em: < http://dx.doi.org/10.1016/j.cvsm.2016.06.011 >.

ZAGER, R. A. Progression Of Acute Kidney Injury To Chronic Kidney Disease: Clinical And Experimental Insights And Queries. Nephron Clinical Practice, v. 127, n. 0, p. 46-50, 2014. ISSN 1660-8151. Disponível em: < http://dx.doi.org/10.1159/000363726 >. 
ZUK, A.; BONVENTRE, J. V. Acute Kidney Injury. Annual Review of Medicine, v. 67, p. 293-307, 2016. ISSN 0066-4219. Disponível em: < http://dx.doi.org/10.1146/annurev-med-050214-013407 >. 